\title{
Order parameter fluctuations in natural time and $b$-value variation before large earthquakes
}

\author{
P. A. Varotsos, N. V. Sarlis, and E. S. Skordas \\ Solid State Section and Solid Earth Physics Institute, Physics Department, University of Athens, \\ Panepistimiopolis, Zografos 157 84, Athens, Greece \\ Correspondence to: P. A. Varotsos (pvaro@otenet.gr)
}

Received: 20 September 2012 - Accepted: 28 October 2012 - Published: 21 November 2012

\begin{abstract}
Self-similarity may stem from two origins: the process increments infinite variance and/or process memory. The $b$-value of the Gutenberg-Richter law comes from the first origin. In the frame of natural time analysis of earthquake data, a fall of the $b$-value observed before large earthquakes reflects an increase of the order parameter fluctuations upon approaching the critical point (mainshock). The increase of these fluctuations, however, is also influenced from the second origin of self-similarity, i.e., temporal correlations between earthquake magnitudes. This is supported by observations and simulations of an earthquake model.
\end{abstract}

\section{Introduction}

A large variety of natural systems exhibit irregular and complex behavior which at first look seems to be erratic, but in fact possesses scale-invariant structure, for example see Peng et al. (1995); Kalisky et al. (2005). A stochastic process $X(t)$ is called self-similar (Lamperti, 1962) with index $H>0$ if it has the property

$X(\lambda t) \stackrel{d}{=} \lambda^{H} X(t) \quad \forall \quad \lambda>0$.

where the equality concerns the finite-dimensional distributions of the process $X(t)$ on the right- and the left-hand side of the equation (not the values of the process).

A point of crucial importance in analyzing data from complex systems that exhibit scale-invariant structure, is the following: in several systems this nontrivial structure stems from long-range temporal correlations; in other words, the self-similarity originates from the process memory only. This is the case for example of fractional Brownian motion. Alternatively, the self-similarity may solely come from the process increments infinite variance. Such an example is Lévy stable motion (the variance of Lévy stable distributions is infinite since they have heavy tails, Weron et al., 2005, thus differing greatly from the Gaussian ones). In general, however, the self-similarity may result from both these origins (Kantelhardt et al., 2002), the presence of which can be in principle identified when analyzing the complex time series in terms of the new time domain termed natural time (Varotsos et al., 2011b).

The evolution of seismicity is a typical example of complex time series. Several traditional studies were focused on the variation of the $b$-value of the Gutenberg-Richter (GR) law (Gutenberg and Richter, 1954), which states that the (cumulative) number of earthquakes with magnitude greater than (or equal to) $M, N(\geq M)$, occurring in a specified area and time is given by

$N(\geq M)=10^{a-b M}$,

where $b$ is a constant, varying only slightly from region to region and the constant $a$ gives the logarithm of the number of earthquakes with magnitude greater than zero (Shcherbakov et al., 2004). These studies found that the $b$-value decreases before a large event, e.g., see Li et al. (1978) (cases where $b$-value increases prior to and then decreases sharply before a large event have been also reported, Henderson and Main, 1992). Here, considering that the $b$-value itself solely focuses on the one origin of self-similarity, and in particular the process increments infinite variance, we show that, when employing natural time analysis, the $b$-value decrease before large earthquakes reflects an increase of the fluctuations of the order parameter of seismicity when approaching the critical point (mainshock, see below). The whole precursory variation of the order parameter fluctuations, however, is more 
complex since it captures both origins. Temporal correlations between earthquake magnitudes also play an important role in this precursory variation, thus leading to additional results compared to the ones obtained when restricting ourselves to traditional analysis of $b$-value alone.

\section{Natural time analysis - identification of the two origins of self-similarity - the case of seismic electric signals activities}

For a time series comprising $N$ events, we define (Varotsos et al., 2001) the natural time $\chi_{k}$ for the occurrence of the $k$-th event (of energy $Q_{k}$ ) by $\chi_{k}=k / N$. We then study the evolution of the pair $\left(\chi_{k}, Q_{k}\right)$ or $\left(\chi_{k}, p_{k}\right)$, where $p_{k}=Q_{k} / \sum_{n=1}^{N} Q_{n}$ is the normalized energy released during the $k$-th event. The quantity $\Phi(\omega)$ is defined by $\Phi(\omega)=$ $\sum_{k=1}^{N} p_{k} \exp \left(i \omega \chi_{k}\right)$, where $\omega$ stands for the natural angular frequency, and then evaluate the real function $\Pi(\omega)=$ $|\Phi(\omega)|^{2}$ in the low frequency limit. By considering the Taylor expansion $\Pi(\omega)=1-\kappa_{1} \omega^{2}+\kappa_{2} \omega^{4}+\ldots$, we find that the approach of a dynamical system to criticality (see Chapter 8 of Varotsos et al., 2011b) is identified by means of $\kappa_{1}$, i.e.,

$\kappa_{1}=\left\langle\chi^{2}\right\rangle-\langle\chi\rangle^{2}=\sum_{k=1}^{N} p_{k} \chi_{k}^{2}-\left(\sum_{k=1}^{N} p_{k} \chi_{k}\right)^{2}$,

which is the variance (Varotsos et al., 2001, 2005, 2011b) of natural time weighted for $p_{k}$. When $Q_{k}$ is independent and identically distributed positive random variables, we obtain the "uniform" (u) distribution of $p_{k}$, as it was defined by Varotsos et al. (2003) (see also p. 122 of Varotsos et al., $2011 b$ ). In this case, all $p_{k}$ vary around their mean value $1 / N$ (cf. since $\sum_{n=1}^{N} p_{n}=1$ ) and the quantity $\kappa_{1}$ results (Varotsos et al., 2003) in $\kappa_{\mathrm{u}}=1 / 12$ for large $N$.

In general, in a complex time series, in order to identify the two origins of self-similarity by means of natural time analysis, we focus on the expectation value $\mathcal{E}\left(\kappa_{1}\right)$ of the variance $\kappa_{1}$ of natural time when sliding a natural time window of length $l$ through a time series of $Q_{k}>0, k=1,2, \ldots N$.

If self-similarity exclusively results from the process memory, the $\mathcal{E}\left(\kappa_{1}\right)$-value should change to $\kappa_{\mathrm{u}}=1 / 12$ for the (randomly) shuffled data. This is the case of the seismic electric signals (SES) activities (Varotsos et al., 1993), which are series of low-frequency $(\leq 1 \mathrm{~Hz})$ electric signals detected a few to several weeks (up to five months) before an earthquake when the stress in the focal region reaches a critical value (and hence long range correlations develop). The criteria according to which the observed electric signals are identified as SES activities have been published by Varotsos and Lazaridou (1991): having installed a multitude of measuring electric dipoles of various lengths and orientations, we verify that the observed electric signals are not induced by small variations of the magnetic field of the Earth due to extra terrestrial sources as well as not originating from nearby man made electrical sources. For example, the three upper channels in Fig. 1b show three SES activities that preceded major earthquakes in southern, southwestern and western Greece, respectively, as depicted in the map of Fig. 1a. For the sake of comparison, the lowest channel shows an SES activity recorded in northern Greece (close to Thessaloniki). In all these four cases, the analysis of their original data lead to $\kappa_{1} \approx 0.07$ (see also below), which turns to $\kappa_{\mathrm{u}}=1 / 12$ upon shuffling the data. On the other hand, if the self-similarity results from process increments infinite variance only, $\mathcal{E}\left(\kappa_{1}\right)$ should be the same (but differing from $\kappa_{\mathrm{u}}$ ) for the original and the (randomly) shuffled data. Finally, when both origins of self-similarity are present, the relative strength of the contribution of the one origin compared to that of the other can be quantified on the basis of Eqs. (12) and (13) of Varotsos et al. (2006b) (see also Varotsos et al., 2011b).

\section{Natural time analysis of seismicity - the order parameter of seismicity}

In what remains, we focus on complex time series of seismicity. Earthquakes exhibit scaling relations chiefly among which is the aforementioned G-R law (Gutenberg and Richter, 1954). For reasons of convenience, we write hereafter G-R law of Eq. (2) in the form $N(\geq M) \propto 10^{-b M}$. Considering that the seismic energy $E$ released during an earthquake is related (Kanamori, 1978) to the magnitude through $E \propto 10^{c M}$, where $c$ is around 1.5, the latter form turns to the distribution,

$P(E) \propto E^{-\gamma}$

where $\gamma=1+b / 1.5$. Hence, $b \approx 1$ means that the exponent $\gamma$ is around $\gamma=1.6$ to 1.7 , see Table 2.1 of Varotsos et al. (2011b).

The complex correlations in time, space and magnitude of earthquakes have been extensively studied (Corral, 2004; Holliday et al., 2006; Eichner et al., 2007; Lippiello et al., 2009; Lennartz et al., 2011; Teisseyre and Górski, 2011). The observed earthquake scaling laws (Turcotte, 1997) seem to indicate the existence of phenomena closely associated with the proximity of the system to a critical point (e.g., see Holliday et al., 2006, and references therein). In the frame of natural time analysis, it has been suggested (Varotsos et al., 2005) (see also pp. 249-254 of Varotsos et al., 2011b) that the order parameter of seismicity is the quantity $\kappa_{1}$. The $\kappa_{1}$-value itself may lead to the determination of the occurrence time of the impending mainshock (Varotsos et al., 2001, 2006a,b, 2011b) when SES data are available. In particular, when the $\kappa_{1}$-value resulting from the natural time analysis of the seismicity subsequent to the SES recording becomes approximately equal to 0.070 , the mainshock occurs within a time window of the order of one week. This has been empirically observed in several cases (Varotsos et al., 2001, 2006a,b) (see also Chapter 7 of Varotsos et al., 2011b) including the three 

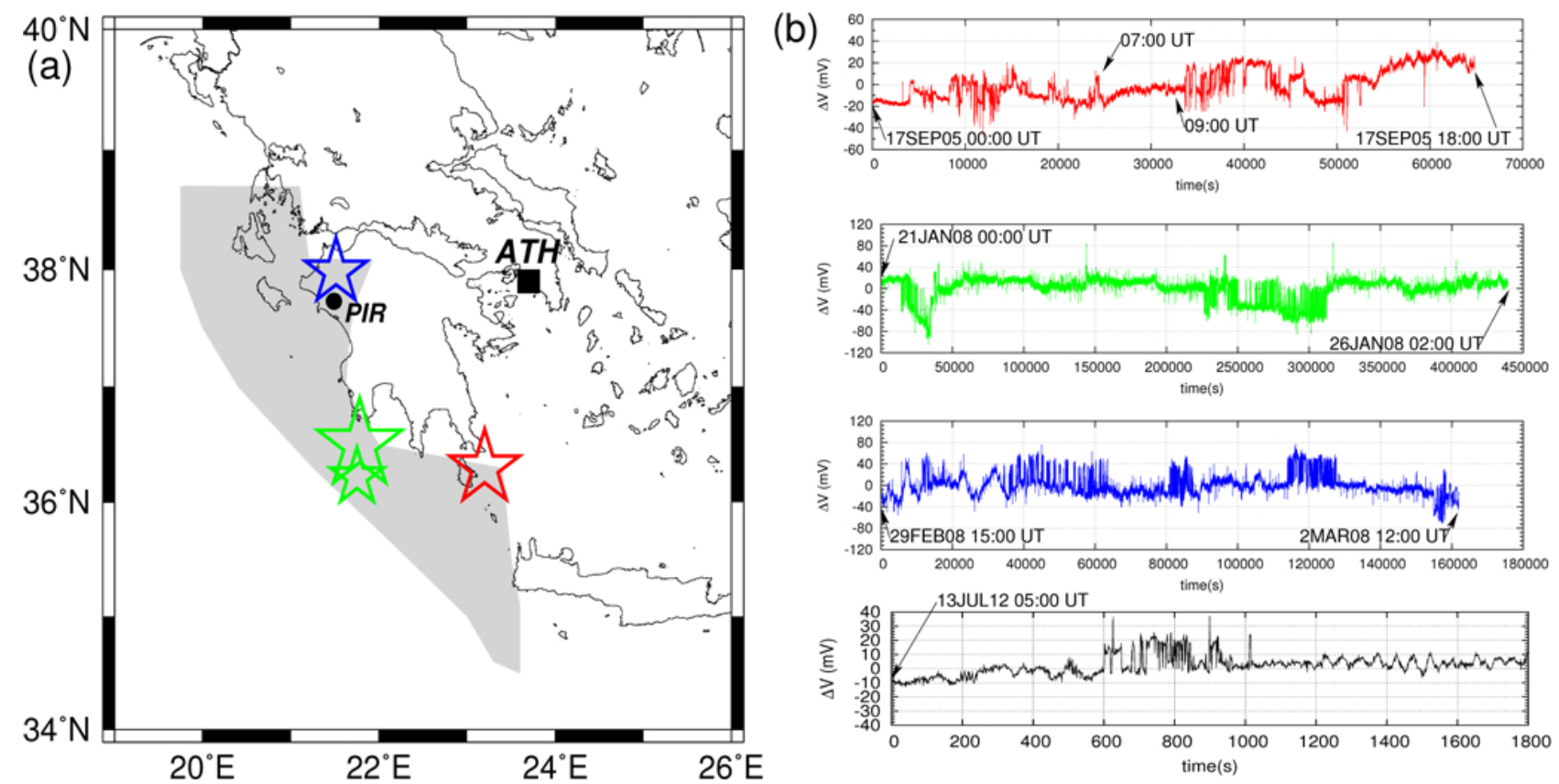

Fig. 1. (color online) (a) Major earthquakes in Greece on 8 January 2006 (red, magnitude $M_{\mathrm{W}}=6.7$ ), 14 February 2008 (green, $M_{\mathrm{W}}=6.9$ and 6.4) and 8 June 2008 (blue, $M_{\mathrm{W}}=6.4$ ). (b) Their preceding SES activities recorded at Pirgos (PIR) measuring station located in western Greece are shown (with the corresponding color) in the upper three channels. Earthquakes with SES activities at PIR are located in the shaded region of (a). Furthermore, an SES activity recorded at a station in northern Greece on 13 July 2012, is depicted in the lowest channel of (b).

major earthquakes of Fig. 1a that followed the SES activities depicted in Fig. 1b. An example of the $\kappa_{1}$ dynamics after the recording of the SES activity depicted in the third channel of Fig. $1 \mathrm{~b}$ until the occurrence of the magnitude 6.4 mainshock on 8 June 2008 (blue star in Fig. 1a) is given in the Appendix (see also Lazaridou-Varotsos, 2012). In the lack of SES data, we have to solely rely on the fluctuations of the order parameter of seismicity. Along these lines, we investigated (Sarlis et al., 2010a) the period before and after a significant mainshock. Time series for various lengths of $W$ earthquakes that occurred before or after the mainshock have been studied. The probability distribution function (pdf) $P\left(\kappa_{1}\right)$ versus $\kappa_{1}$ was found to exhibit a bimodal feature when approaching a mainshock. To quantify this feature, we considered the variability of $\kappa_{1}$, which is just the ratio

$\beta \equiv \sigma\left(\kappa_{1}\right) / \mu\left(\kappa_{1}\right)$,

where $\sigma\left(\kappa_{1}\right)$ and $\mu\left(\kappa_{1}\right)$ stand for the standard deviation and the mean value of $\kappa_{1}$ for sliding window lengths $l=$ 6-40. The bimodal feature reflects that, upon approaching the mainshock (with the number $W$ of the earthquakes before mainshock decreasing), the variability of $\kappa_{1}$ should increase. This was subsequently confirmed because before the $M=9.0$ devastating Tohoku earthquake in Japan on 11 March 2011, the variability of $\kappa_{1}$ exhibited (Uyeda and Varotsos, 2011; see also pp. 207-217 of Lazaridou-Varotsos, 2012; Varotsos et al., 2012) a dramatic increase.
In addition, we investigated (Varotsos et al., 2011a) the order parameter fluctuations, but when considering a natural time window of a fixed-length $W$ sliding through a seismic catalog (cf. in general the results of complexity measures when considering $W=$ const complement (Varotsos et al., 2011b) those deduced when taking windows of various lengths $W$ in the following sense: if in the frame of the one type of measures an ambiguity emerges in identifying the approach to the critical point, the other type gives a very clear answer). For earthquakes in California and Greece, we found (Varotsos et al., 2011a) that when $W$ becomes compatible with the lead time of the SES activities (i.e., of the order of a few months), the fluctuations exhibit a global minimum before the strongest mainshock that occurred during a $25-$ and $10-y r$ period, respectively.

\section{Interrelation between the fluctuations of the order pa- rameter of seismicity and the $b$-value of the G-R law}

Let us now study the interrelation between the $b$-value and the variability of $\kappa_{1}$. In particular, we investigate the expected value of $\kappa_{1}$ when a natural time window length is sliding through randomly shuffled power law distributed energy bursts that obey Eq. (4). In Fig. 2, the pdf $P\left(\kappa_{1}\right)$ versus $\kappa_{1}$ is plotted for several $b$-values, an inspection of which reveals that: For high $b$-values, e.g., for $b=1.5$ and 1.4, the $P\left(\kappa_{1}\right)$ versus $\kappa_{1}$ curve is almost unimodal maximizing at a value 


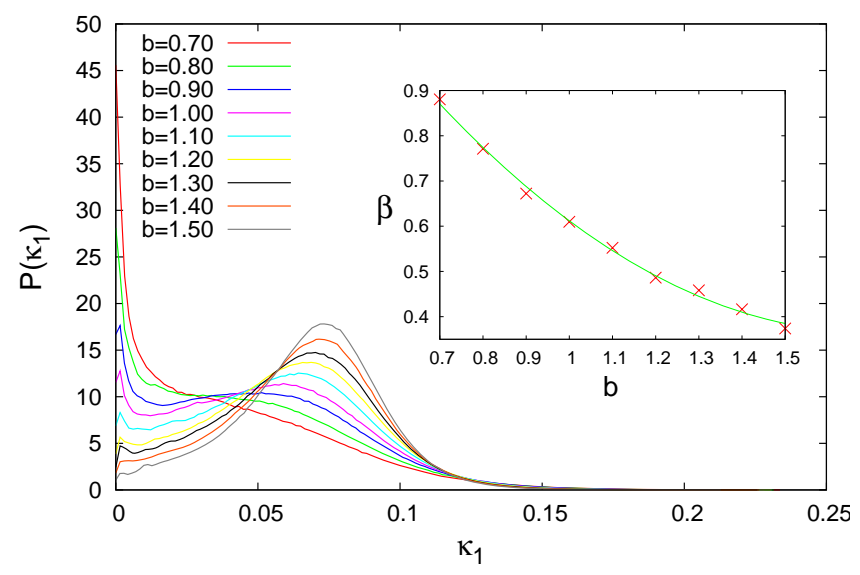

Fig. 2. (color online) The probability density function $P\left(\kappa_{1}\right)$ versus $\kappa_{1}$ for several values of $b$ for temporally uncorrelated events obeying Eq. (4). The inset depicts the variability $\beta$ as a function of $b$ (the cross symbols refer to directly computed values, while the curve has been drawn as a guide to the eye).

somewhat larger than 0.070 , while for smaller $b$ a second mode emerges close to $\kappa_{1} \approx 0$ which reflects that the fluctuations of $\kappa_{1}$ are larger. The computed values of the $\kappa_{1}$ variability as a function of the $b$-value are plotted in the inset of Fig. 2b. The general feature of this curve is more or less similar to that observed for example before Tohoku earthquake (Varotsos et al., 2012); quantitative agreement cannot be demanded, however, because temporal correlations between the earthquake magnitudes are also present which influence the observed results. This is corroborated by the following results obtained from the Olami-Feder-Christensen (OFC) earthquake model (Olami et al., 1992). We preferred to employ this model here, since it has been studied in detail in hundreds of publications, but we clarify that there exist more recent ones, e.g., see Dieterich and Richards-Dinger (2010) where the primary role of the fault system geometry is emerged.

Before proceeding to the presentation of our results obtained from the OFC model, we note that concerning the correlations between magnitudes of subsequent earthquakes, there is a diversity of views in the literature. Such correlations, reported by Lippiello et al. (2007, 2008), have been later attributed (Davidsen and Green, 2011; Davidsen et al., 2012) to catalog incompleteness. In a subsequent publication, however, Lippiello et al. (2012) made an analysis of two California regions with different levels of catalog accuracy and different lower magnitude thresholds, which convincingly indicated that the amplitude of correlations does $n o t$ depend on catalog incompleteness. Natural time analysis of seismicity (Varotsos et al., 2006b, 2011b; Sarlis et al., $2009,2010 \mathrm{~b})$ leads to results that conform to the view expressed by Lippielo and coworkers.

The OFC model runs as follows: we assign a continuous random variable $z_{i j} \in(0,1)$ to each site of a square lattice, which represents the local "energy". Starting with a random initial configuration taken from a uniform distribution in the segment $(0,1)$, the value $z_{i j}$ of all sites is simultaneously increased at a uniform loading rate until a site $i j$ reaches the threshold value $z_{\text {thres }}=1$ (i.e., the loading $\Delta f$ is such that $\left.\left(z_{i j}\right)_{\max }+\Delta f=1\right)$. This site then topples which means that $z_{i j}$ is reset to zero and an "energy" $\alpha z_{i j}$ is passed to every nearest neighbor, where the coupling parameter $\alpha$ can take values from zero to 0.25 and is the only parameter of the model, apart from the edge length $L$ of the square lattice. If this causes a neighbor to exceed the threshold, the neighbor topples also, and the avalanche continues until all $z_{k l}<1$. Then the uniform loading increase resumes. The number of topplings defines the size of an avalanche or "earthquake" and (when it is larger than unity $k$ increases by one) is used as $Q_{k}$ in natural time analysis. Here, we use the case of free boundary conditions (Helmstetter et al., 2004) in which $\alpha$ varies locally $\alpha_{i j}=\frac{1}{n_{i j}+K}$, where $n_{i j}$ is the actual number of nearest neighbors of the site $i j$ (for sites in the bulk $n_{i j}=4$, for sites at the edges $n_{i j}=3$ and for the four sites at the corners $n_{i j}=2$ ) and $K$ denotes (Helmstetter et al., 2004) the elastic constant of the upper leaf springs measured relatively to that of the other springs between blocks in the Burridge-Knopoff model (Burridge and Knopoff, 1967). The OFC model is obviously non-conservative for $K>0$ for which $\alpha_{i j}<0.25$ in the bulk (for more details on the OFC modeling see pp. 349-363 of Varotsos et al., 2011b and references therein).

We first study whether there exists predictability in the OFC model on the basis of the $\kappa_{1}$ variability. In other words, we study whether the probability for the occurrence of a large avalanche differs from that of random chance when the sequential order of the earlier avalanches has led to a value of the $\kappa_{1}$ variability that exceeds some threshold (see below). We consider the variability $\beta_{k}$ which is a function of the natural time index $k, k=1,2, \ldots, N=2 \times 10^{6}$ estimated by analyzing in natural time for each $k$ the preceding $W=100$ avalanches. The time increased probability (TIP) (Keilis-Borok and Rotwain, 1990) (i.e., the time during which there exists a high probability for the occurrence of a large avalanche exceeding a given threshold) is turned on when $\beta_{k}>\beta_{c}$, where $\beta_{c}$ is a given threshold in the prediction. If the size $Q_{k}$ is greater than a target avalanche size threshold $Q_{c}$, we have a successful prediction. For binary predictions, the prediction of events becomes a classification task with two types of errors: missing an event and giving a false alarm. We therefore choose (Garber et al., 2009) the receiver operating characteristics (ROC) graph (Fawcett, 2006) to depict the prediction quality. This is a plot of the hit rate versus the false alarm rate, as a function of the total rate of alarms, which here is tuned by the threshold $\beta_{c}$. Only if in between the hit rate exceeds the false alarm rate, the predictor is useful. Random predictions generate equal hit and alarm rate, and hence they lead to the diagonal in ROC plot. 


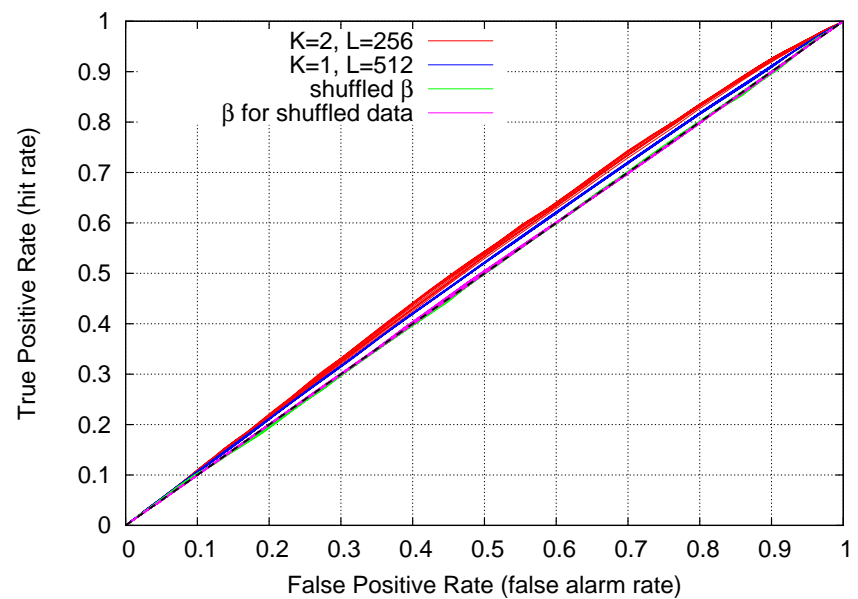

Fig. 3. (color online) The ROC diagram for the OFC earthquake model discussed in the text: $\operatorname{red}(L=256$ and $K=2)$ and blue $(L=$ 512 and $K=1)$ lines. In addition, two ROC diagrams are depicted based on the results obtained for $L=512$ and $K=1$ : the green curves correspond to the case when the values of $\beta_{k}$ were randomly shuffled and the shuffled predictors were used, while the magenta curves when the time series of $Q_{k}$ was randomly shuffled and then $\beta_{k}$ was estimated.

Thus, only when the points lie above this diagonal the predictor is useful. As an example, the ROC graphs for $L=512$ and $K=1$ or $L=256$ and $K=2$ are shown in Fig. 3 (the rational for choosing these two cases stems from the study of Lise and Paczuski (2001) in which it was shown that the OFC model with free boundary conditions exhibits in these cases - see their Fig. 4 - avalanche size distribution that agrees with the G-R law). For every given threshold value $\beta_{c}$ and a target threshold $Q_{c}$, we get a point in this plot, thus varying $\beta_{c}$ we get a curve. The various curves in Fig. 3 correspond to various values of $Q_{c}=168, \ldots, 1000$ increasing from the bottom to the top. An inspection of this figure shows that the points in each curve lie above the diagonal and the excess is higher for larger values of $Q_{c}$. In order to investigate the statistical validity of this result, we include in the same graph the results where: (a) the values of $\beta_{k}$ were randomly shuffled and the shuffled predictors were used (green curves) and (b) the time series of $Q_{k}$ was randomly shuffled and then $\beta_{k}$ was estimated (magenta curves); in both cases, we obtain curves which almost coincide with the diagonal. This clearly demonstrates that the aforementioned excess of the results related with the original $Q_{k}$ series from the diagonal comes from the sequential order of avalanches and cannot be considered as chancy.

We now proceed to the investigation of the temporal correlations between the magnitudes $m_{k}=\log _{10}\left(Q_{k}\right) / 1.5 \mathrm{ob}-$ tained from the sizes $Q_{k}$ of the avalanches in the OFC model preceding a large avalanche. The results can be visualized in two examples in Fig. 4, where we plot in blue the exponent $a_{\mathrm{DFA}}$ of the detrended fluctuation analysis (DFA)
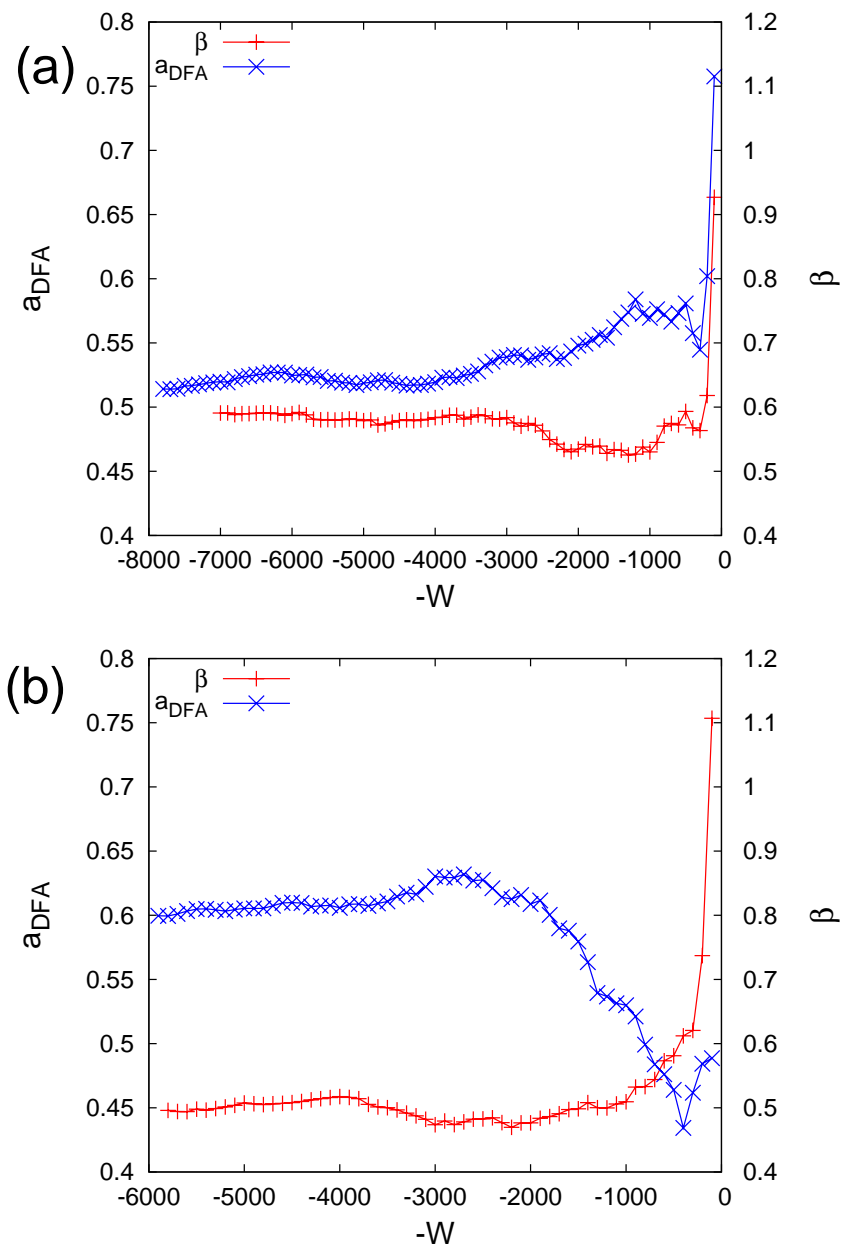

Fig. 4. (color online) The exponent $a_{\mathrm{DFA}}$ (blue, left scale) and the variability $\beta$ (red, right scale) versus the number of the avalanches preceding a large avalanche, $Q_{k}=40325$ for (a) and $Q_{k}=31145$ for (b), that corresponds to $W=0$ for the OFC model ( $K=1, L=$ 512).

(Peng et al., 1994) (along with the variability $\beta$ plotted in red) versus the number $W$ of avalanches before a large avalanche (negative $\mathrm{x}$ semi-axis, $x=-W$ ). Note that DFA has already been employed by Livina and Lenton (2007) for monitoring temporal correlations before bifurcations. In the upper example, Fig. $4 \mathrm{a}$, the value of $a_{\mathrm{DFA}}$ well before the large avalanche, being somewhat larger than 0.5 , exhibits small changes but strongly increases upon approaching the large avalanche, i.e., at $W=100$ the value of $a_{\text {DFA }}$ becomes $\approx 0.75$ which shows intensified temporal correlations. In the lower example, Fig. $4 \mathrm{~b}$, well before the large avalanche we have $a_{\mathrm{DFA}} \approx 0.6$ showing long range temporal correlations, which first turn to anti-correlations upon approaching the large avalanche, e.g., $a_{\mathrm{DFA}} \approx 0.43$ at $W=400$, and finally become random, i.e, $a_{\mathrm{DFA}} \approx 0.5$ at $W=100$, just before the "mainshock". In both examples of Fig. 4, the variability $\beta$ rapidly increases upon approaching a large 
avalanche showing clear precursory changes in the temporal correlations between avalanche magnitudes. A detailed statistical study of the OFC model $(K=1, L=512)$, for $W=$ $100,200, \ldots 1000$, showed that among the 579 large $\left(Q_{k}>\right.$ 30000 ) avalanches, in $30 \%$ of the cases a rapid increase of $\beta$ upon approaching them is observed. This is more or less consistent with empirical observations since in Japan this precursory increase was observed in 8 out of 25 earthquakes (all above $M=7$ during 1 January 1994 to 11 March 2011 with depths smaller than $700 \mathrm{~km}$ ) (Varotsos et al., 2012). Concerning the $\alpha$-values, when studying $W=100,200, \ldots 1000$, among the 579 large avalanches studied, in $76 \%$ of the cases the $\alpha$-value was found to become smaller than 0.5 (as seen in Fig. 4b).

\section{Summary and conclusions}

Traditional analyses of seismicity make use of the $b$-value itself, which focuses on the one origin of self-similarity, i.e., the process increments infinite variance. Here, in order to shed light on the long standing observation that the $b$-value occasionally decreases before large earthquakes, we employ natural time analysis, which is a general procedure to analyze complex time series without the need to introduce any assumption and/or adjustable parameter(s). Our main finding constitutes in identifying, for the first time, that the variation of the $b$-value is interconnected with the fluctuations of the order parameter $\kappa_{1}$ of seismicity when approaching the critical point (mainshock). In particular:

1. We show that, in general, for randomly shuffled powerlaw distributed data (see Eq. 4), the $b$-value decrease reflects an increase of the variability $\beta$ of the order parameter $\kappa_{1}$, as shown in the inset of Fig. 2.

2. An experimentally observed increase of the variability $\beta$ before a large earthquake, however, cannot be arbitrarily attributed to the $b$-value decrease alone, mentioned in point 1 , because the other origin of self-similarity, i.e., temporal correlations between earthquake magnitudes, may also be present and influence the effect observed. To quantify the relative strength of the contribution of the one origin of self-similarity compared to that of the other, the procedure developed in Sarlis et al. (2009) (based on Eqs. (12) and (13) of Varotsos et al., 2006b) must be employed by analyzing in natural time the data available in each case.

3. Although it cannot capture all the characteristics of earthquake dynamics, the OFC model was employed here to investigate the existence of correlations between the magnitudes obtained from the sizes of the avalanches preceding a large avalanche. We find that this holds beyond chance arising from the sequential order of avalanches. In other words, we show that the probability for the occurrence of a large avalanche exceeds that of random chance when the sequential order of the earlier avalanches has resulted in a relatively high value of the variability $\beta$. In addition, examples are presented in which the variability $\beta$ increases upon approaching a large avalanche showing precursory changes in the temporal correlations between avalanche magnitudes. In the specific OFC model studied, we found that among the largest 579 avalanches, in $30 \%$ of the cases a rapid $\beta$ increase was observed until just before the occurrence of a large avalanche.

\section{Appendix A}

A tentative procedure to identify the occurrence time of an impending mainshock: the case of the $M_{\mathrm{w}}=6.4$ earthquake on 8 June 2008

Upon the recording of an SES activity, one can estimate (through the procedure explained in Chapter 1 of Varotsos et al., 2011b) an area A within which the impending main shock is expected to occur. We then analyze in natural time the subsequent seismicity (as it evolves event by event) in all the possible subareas of A. The $\kappa_{1}$-values of all these subareas and the largest area A, are treated on equal footing and allow the construction of the probability distribution $\operatorname{Prob}\left(\kappa_{1}\right)$. This way $\operatorname{Prob}\left(\kappa_{1}\right)$ versus $\kappa_{1}$ is obtained until it maximizes at $\kappa_{1} \approx 0.070$ exhibiting also magnitude threshold invariance. This, according to our observations to date, usually occurs a few days to around one week or so before the mainshock, thus it gives the possibility to estimate the occurrence time of major earthquakes. Example of this procedure will now be presented for the earthquake (EQ) of magnitude $M_{\mathrm{w}}=6.4$ that occurred in Greece on 8 June 2008.

This major EQ was preceded by the SES activity that lasted from 29 February to 2 March 2008, which is shown in the third channel of Fig. 1b. After subtracting the sinusoidal background noise, termed magnetotelluric (arising from electric field variations on the Earth's surface induced by the frequent variations of the Earth's magnetic field) with the procedure described in Varotsos et al. (2009), the signal was analyzed in natural time and classified as an SES activity.

The investigation of the subsequent seismicity was conducted at first (see Sarlis et al., 2008a) in the area $A: N_{37.0}^{38.6} E_{20.0}^{22.0}$, which is somewhat smaller than the PIR selectivity map (i.e., the map containing the seismic areas which emit precursory SES recorded at PIR measuring station) known at that time. This was in an attempt to avoid as much as possible the influence of aftershocks of the $M_{\mathrm{w}}=6.9 \mathrm{EQ}$ at $36.5^{\circ} \mathrm{N} 21.8^{\circ} \mathrm{E}$ on 14 February 2008. This policy was considered justified, based on the notion that a criticality approach would take place in proper subareas simultaneously. At the same time, an attempt was also made 

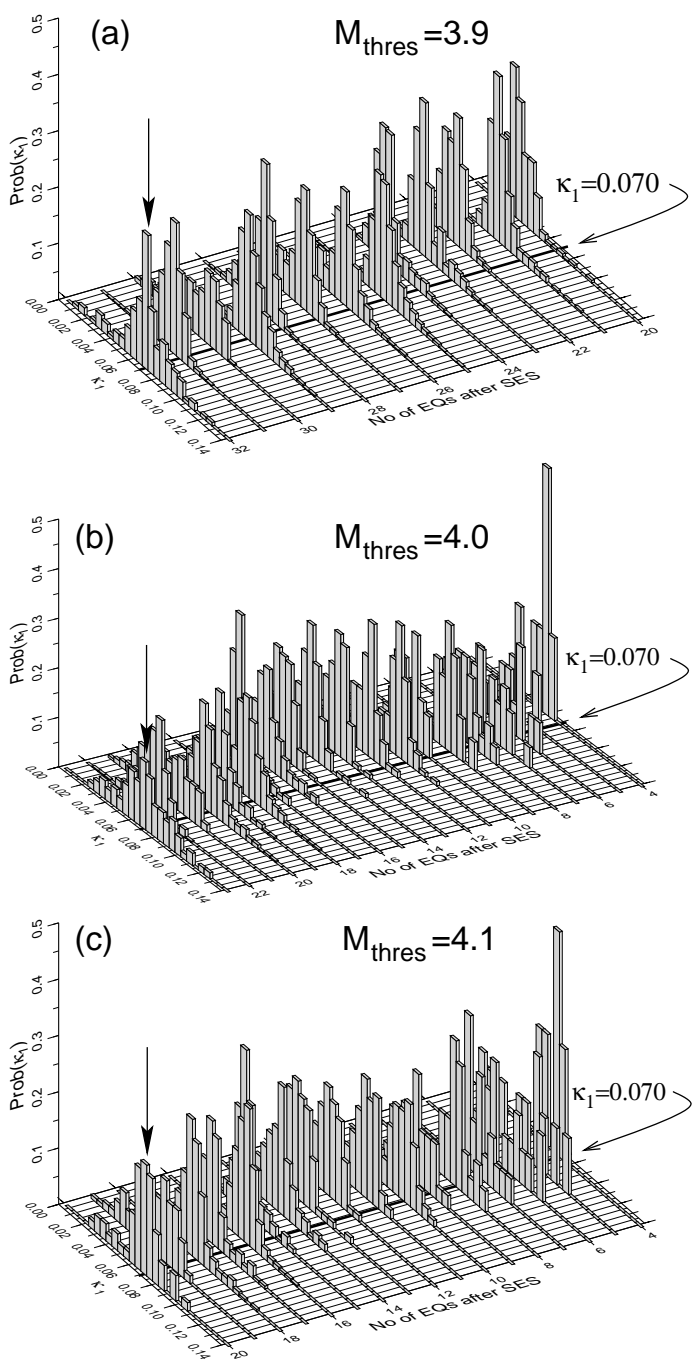

Fig. A1. The probability $\operatorname{Prob}\left(\kappa_{1}\right)$ versus $\kappa_{1}$ of the seismicity with magnitude threshold $M_{\text {thres }}=3.9$ (a), $M_{\text {thres }}=4.0$ (b) and $M_{\text {thres }}=4.1$ (c) within the shaded area shown in Fig. 1a subsequent to the SES activity recorded at PIR during 29 February to 2 March 2008, see the third channel in Fig. 1b. The vertical arrows mark the maxima of $\operatorname{Prob}\left(\kappa_{1}\right)$ versus $\kappa_{1}$ at $\kappa_{1} \approx 0.070$ that occurred at 23:26 UT on 27 May 2008 (practically 28 May) and has been followed by the $M_{\mathrm{W}}=6.4$ on 8 June 2008. Taken from Sarlis et al. (2008c).

to extend the area A to include the shaded area shown in Fig. 1a. This extension was based on the recent - at that time - pieces of information for PIR selectivity map, including the occurrences of the aforementioned $M_{\mathrm{W}}=6.9 \mathrm{EQ}$ on 14 February 2008 associated with the SES activity depicted in the second channel of Fig. $1 \mathrm{~b}$ and the $M_{\mathrm{w}}=6.7 \mathrm{EQ}$ at $36.3^{\circ} \mathrm{N} 23.2^{\circ} \mathrm{E}$ on 8 January 2006 following the SES activity of the upper channel of the same figure (details on the latter event have been described in Varotsos, 2006). In the study for the extended PIR selectivity map area (shaded region in Fig. 1a), we raised the magnitude threshold to
$M_{\text {thres }}=3.9,4.0$ and 4.1 (local magnitudes $M_{\mathrm{L}}$ ), because the extended area along the Hellenic Arc was highly seismic and there were too many (more than half a thousand) events to handle for $M_{\text {thres }}=3.2$. This study showed that upon the occurrence of a $M_{\mathrm{S}}(\mathrm{ATH})=5.1 \mathrm{EQ}$ (where $M_{\mathrm{S}}(\mathrm{ATH})$ stands for the $M_{\mathrm{S}}$ magnitude announced by the Athens Observatory, ATH, $\left.M_{\mathrm{S}}(\mathrm{ATH})=M_{\mathrm{L}}+0.5\right)$ at $35.5^{\circ} \mathrm{N} 22.4^{\circ} \mathrm{E}$ at 23:26 UT on 27 May (practically 28 May) 2008, the probability $\operatorname{Prob}\left(\kappa_{1}\right)$ exhibited a pronounced maximum at $\kappa_{1} \approx 0.070$ marked by a vertical arrow in Fig. A1a drawn for $M_{\text {thres }}=3.9$. Similar maxima at $\kappa_{1} \approx 0.070$ appeared simultaneously for $M_{\text {thres }}=4.0$ and $M_{\text {thres }}=4.1$ (see Fig. A1b and c, respectively), thus indicating that the critical point has been approached. This was reported on 29 May 2008 in Sarlis et al. (2008c) (see also Sarlis et al., 2008b; Lazaridou-Varotsos, 2012). Actually, almost 10 days later, i.e., at 12:25 UT on 8 June 2008 , a $M_{\mathrm{W}}=6.4 \mathrm{EQ}$ occurred at $38.0^{\circ} \mathrm{N} 21.5^{\circ} \mathrm{E}$, i.e., inside the shaded area shown in Fig. 1a, which caused extensive damage.

Edited by: M. E. Contadakis

Reviewed by: two anonymous referees

\section{References}

Burridge, R. and Knopoff, L.: Model and theoretical seismicity, Bull. Seismol. Soc. Am., 57, 341-371, 1967.

Corral, A.: Long-Term Clustering, Scaling, and Universality in the Temporal Occurrence of Earthquakes, Phys. Rev. Lett., 92, 108501, doi:10.1103/PhysRevLett.92.108501, 2004.

Davidsen, J. and Green, A.: Are Earthquake Magnitudes Clustered?, Phys. Rev. Lett., 106, 108502, doi:10.1103/PhysRevLett.106.108502, 2011.

Davidsen, J., Kwiatek, G., and Dresen, G.: No Evidence of Magnitude Clustering in an Aftershock Sequence of Nano- and Picoseismicity, Phys. Rev. Lett., 108, 038501, doi:10.1103/PhysRevLett.108.038501, 2012.

Dieterich, J. H. and Richards-Dinger, K. B.: Earthquake Recurrence in Simulated Fault Systems, Pure Appl. Geophys., 167, 1087, doi:10.1007/s00024-010-0094-0, 2010.

Eichner, J. F., Kantelhardt, J. W., Bunde, A., and Havlin, S.: Statistics of return intervals in long-term correlated records, Phys. Rev. E, 75, 011128, doi:10.1103/PhysRevE.75.011128, 2007.

Fawcett, T.: An introduction to ROC analysis, Pattern Recogn. Lett., 27, 861-874, 2006.

Garber, A., Hallerberg, S., and Kantz, H.: Predicting extreme avalanches in self-organized critical sandpiles, Phys. Rev. E, 80, 026124, doi:10.1103/PhysRevE.80.026124, 2009.

Gutenberg, B. and Richter, C. F.: Seismicity of the Earth and Associated Phenomena, Princeton Univ. Press, Princeton, New York, 1954.

Helmstetter, A., Hergarten, S., and Sornette, D.: Properties of foreshocks and aftershocks of the nonconservative self-organized critical Olami-Feder-Christensen model, Phys. Rev. E, 70, 046120, doi:10.1103/PhysRevE.70.046120, 2004.

Henderson, J. and Main, I.: A simple fracture-mechanical model for the evolution of seismicity, Geophys. Res. Lett., 19, 365-368, 
1992.

Holliday, J. R., Rundle, J. B., Turcotte, D. L., Klein, W., Tiampo, K. F., and Donnellan, A.: Space-Time Clustering and Correlations of Major Earthquakes, Phys. Rev. Lett., 97, 238501, doi:10.1103/PhysRevLett.97.238501, 2006.

Kalisky, T., Ashkenazy, Y., and Havlin, S.: Volatility of linear and nonlinear time series, Phys. Rev. E, 72, 011913, doi:10.1103/PhysRevE.72.011913, 2005.

Kanamori, H.: Quantification of Earthquakes, Nature, 271, 411414, 1978.

Kantelhardt, J. W., Ashkenazy, Y., Ivanov, P. C., Bunde, A., Havlin, S., Penzel, T., Peter, J.-H., and Stanley, H. E.: Characterization of sleep stages by correlations in the magnitude and sign of heartbeat increments, Phys. Rev. E, 65, 051908, doi:10.1103/PhysRevE.65.051908, 2002.

Keilis-Borok, V. I. and Rotwain, I. M.: Diagnosis of Time of Increased Probability of strong earthquakes in different regions of the world: algorithm CN, Phys. Earth Planet. Inter., 61, 57-72, 1990.

Lamperti, J. W.: Semi-stable stochastic processes, Trans. Am. Math. Soc., 104, 62-78, 1962.

Lazaridou-Varotsos, M. S.: Earthquake Prediction by Seismic Electric Signals: The success of the VAN method over thirty years, Springer Praxis Books, Berlin Heidelberg, 2012.

Lennartz, S., Bunde, A., and Turcotte, D. L.: Modelling seismic catalogues by cascade models: Do we need long-term magnitude correlations?, Geophys. J. Int., 184, 1214-1222, 2011.

Li, Q., Cen, J., Yu, L., and Hao, B.: Time and space scanning of the b-value - a method for monitoring the developement of catastrophic earthquakes, Acta Geophys. Sinica, 21, 101-125, 1978.

Lippiello, E., Godano, C., and de Arcangelis, L.: Dynamical Scaling in Branching Models for Seismicity, Phys. Rev. Lett., 98, 098501, doi:10.1103/PhysRevLett.98.098501, 2007.

Lippiello, E., de Arcangelis, L., and Godano, C.: Influence of Time and Space Correlations on Earthquake Magnitude, Phys. Rev. Lett., 100, 038501, doi:10.1103/PhysRevLett.100.038501, 2008.

Lippiello, E., de Arcangelis, L., and Godano, C.: Role of Static Stress Diffusion in the Spatiotemporal Organization of Aftershocks, Phys. Rev. Lett., 103, 038501, doi:10.1103/PhysRevLett.103.038501, 2009.

Lippiello, E., Godano, C., and de Arcangelis, L.: The earthquake magnitude is influenced by previous seismicity, Geophys. Res. Lett., 39, L05309, doi:10.1029/2012GL051083, 2012.

Lise, S. and Paczuski, M.: Self-organized criticality and universality in a nonconservative earthquake model, Phys. Rev. E, 63, 036111, doi:10.1103/PhysRevE.63.036111, 2001.

Livina, V. N. and Lenton, T. M.: A modified method for detecting incipient bifurcations in a dynamical system, Geophys. Res. Lett., 34, L03712, doi:10.1029/2006GL028672, 2007.

Olami, Z., Feder, H. J. S., and Christensen, K.: Self-organized criticality in a continuous, nonconservative cellular automaton modeling earthquakes, Phys. Rev. Lett., 68, 1244-1247, 1992.

Peng, C.-K., Buldyrev, S. V., Havlin, S., Simons, M., Stanley, H. E., and Goldberger, A. L.: Mosaic organization of DNA nucleotides, Phys. Rev. E, 49, 1685-1689, 1994.

Peng, C. K., Buldyrev, S. V., Goldberger, A. L., Havlin, S., Mantegna, R. N., Simons, M., and Stanley, H. E.: Statistical properties of DNA sequences, Physica A, 221, 180-192, 1995.
Sarlis, N. V., Skordas, E. S., Lazaridou, M. S., and Varotsos, P. A.: Investigation of the seismicity after the initiation of a Seismic Electric Signal activity until the main shock, arXiv:0802.3329v2 [cond-mat.stat-mech], 20 March 2008a.

Sarlis, N. V., Skordas, E. S., Lazaridou, M. S., and Varotsos, P. A.: Investigation of seismicity after the initiation of a Seismic Electric Signal activity until the main shock, Proc. Japan Acad. Ser. B, 84, 331-343, 2008b.

Sarlis, N. V., Skordas, E. S., Lazaridou, M. S., and Varotsos, P. A.: Investigation of the seismicity after the initiation of a Seismic Electric Signal activity until the main shock, arXiv:0802.3329v4 [cond-mat.stat-mech], 29 May 2008c.

Sarlis, N. V., Skordas, E. S., and Varotsos, P. A.: Multiplicative cascades and seismicity in natural time, Phys. Rev. E, 80, 022102, doi:10.1103/PhysRevE.80.022102, 2009.

Sarlis, N. V., Skordas, E. S., and Varotsos, P. A.: Order parameter fluctuations of seismicity in natural time before and after mainshocks, EPL, 91, 59001, doi:10.1209/0295-5075/91/59001, 2010a.

Sarlis, N. V., Skordas, E. S., and Varotsos, P. A.: Nonextensivity and natural time: The case of seismicity, Phys. Rev. E, 82, 021110, doi:10.1103/PhysRevE.82.021110, 2010b.

Shcherbakov, R., Turcotte, D. L., and Rundle, J. B.: A generalized Omori's law for earthquake aftershock decay, Geophys. Res. Lett., 31, L11613, doi:10.1029/2004GL019808, 2004.

Teisseyre, R. and Górski, M.: Earthquake Fragmentation and Slip Processes: Spin and Shear-Twist Wave Mosaic, Acta Geophys., 59, 453-469, 2011.

Turcotte, D. L.: Fractals and Chaos in Geology and Geophysics, Cambridge University Press, Cambridge, 2nd Edn., 1997.

Uyeda, S. and Varotsos, P.: Earthquake Prediction in Japan and Natural Time Analysis of Seismicity (Invited), U23C-03, presented at 2011 Fall meeting, AGU, San Francisco, Calif., 5-8 December 2011.

Varotsos, P. A.: Recent Seismic Electric Signals (SES) activities in Greece, Acta Geophys. Pol., 54, 158-164, 2006.

Varotsos, P. and Lazaridou, M.: Latest aspects of earthquake prediction in Greece based on Seismic Electric Signals, Tectonophysics, 188, 321-347, 1991.

Varotsos, P., Alexopoulos, K., and Lazaridou, M.: Latest aspects of earthquake prediction in Greece based on Seismic Electric Signals, II, Tectonophysics, 224, 1-37, 1993.

Varotsos, P. A., Sarlis, N. V., and Skordas, E. S.: Spatio-Temporal complexity aspects on the interrelation between Seismic Electric Signals and Seismicity, Practica of Athens Academy, 76, 294 321, 2001.

Varotsos, P. A., Sarlis, N. V., and Skordas, E. S.: Longrange correlations in the electric signals the precede rupture: Further investigations, Phys. Rev. E, 67, 021109, doi:10.1103/PhysRevE.67.021109, 2003.

Varotsos, P. A., Sarlis, N. V., Tanaka, H. K., and Skordas, E. S.: Similarity of fluctuations in correlated systems: The case of seismicity, Phys. Rev. E, 72, 041103, doi:10.1103/PhysRevE.72.041103, 2005.

Varotsos, P. A., Sarlis, N. V., Skordas, E. S., Tanaka, H. K., and Lazaridou, M. S.: Entropy of seismic electric signals: Analysis in the natural time under time reversal, Phys. Rev. E, 73, 031114, doi:10.1103/PhysRevE.73.031114, 2006a. 
Varotsos, P. A., Sarlis, N. V., Skordas, E. S., Tanaka, H. K., and Lazaridou, M. S.: Attempt to distinguish long-range temporal correlations from the statistics of the increments by natural time analysis, Phys. Rev. E, 74, 021123, doi:10.1103/PhysRevE.74.021123, 2006b.

Varotsos, P. A., Sarlis, N. V., and Skordas, E. S.: Detrended fluctuation analysis of the magnetic and electric field variations that precede rupture, CHAOS, 19, 023114, doi:10.1063/1.3130931, 2009.

Varotsos, P., Sarlis, N., and Skordas, E.: Scale-specific order parameter fluctuations of seismicity in natural time before mainshocks, EPL, 96, 59002, doi:10.1209/0295-5075/96/59002, 2011a.

Varotsos, P. A., Sarlis, N. V., and Skordas, E. S.: Natural Time Analysis: The new view of time. Precursory Seismic Electric Signals, Earthquakes and other Complex Time-Series, Springer-Verlag, Berlin Heidelberg, 2011b.
Varotsos, P. A., Sarlis, N. V., Skordas, E. S., Uyeda, S., Kamogawa, M., Nagao, T., and Tanaka, H.: Fluctuations of an order parameter for seismicity before the 1995 M7.3 Kobe and the 2011 M9.0 Tohoku earthquakes, Proc. Jpn. Acad., Ser. B, to be published, 2012.

Weron, A., Burnecki, K., Mercik, S., and Weron, K.: Complete description of all self-similar models driven by Lévy stable noise, Phys. Rev. E, 71, 016113, doi:10.1103/PhysRevE.71.016113, 2005. 